(2) Open Access Full Text Article

\title{
Association of systolic blood pressure drop with intravenous administration of itraconazole in children with hemato-oncologic disease
}

This article was published in the following Dove Press journal:

Drug Design, Development and Therapy

17 December 2015

Number of times this article has been viewed

\author{
Hyeong Jin Lee ${ }^{1, *}$ \\ Bongjin Lee ${ }^{2, *}$ \\ June Dong Park ${ }^{2}$ \\ Hyung Joo Jeong ${ }^{2}$ \\ Yu Hyeon $\mathrm{Choi}^{2}$ \\ Hee Young Ju' \\ Che Ry Hong' \\ JiWon Lee' \\ Hyery Kim' \\ Dong In Suh ${ }^{3}$ \\ Kyung Duk Park' \\ Hyoung Jin Kang' \\ Hee Young Shin' \\ Hyo Seop Ahn'
}

'Department of Pediatrics, Cancer Research Institute, ${ }^{2}$ Division of Pediatric Intensive Care,

Department of Pediatrics, ${ }^{3}$ Division of Pulmonology, Department of

Pediatrics, Seoul National University College of Medicine, Seoul National

University, Seoul, South Korea

*These authors contributed equally to this work

Correspondence: June Dong Park Division of Pediatric Intensive Care, Department of Pediatrics, Seoul National University College of Medicine, Seoul National University, I0I Daehak-no, Jongno-gu, Seoul 03080, South Korea $\mathrm{Tel}+82220723359$

Fax +82 27623359

Email jdparkmd@snu.ac.kr
Purpose: Although few adverse effects have been reported for itraconazole, a widely used antifungal therapy for febrile neutropenia, we found intravenous (IV) itraconazole to be associated with serious cases of blood pressure (BP) drop. We therefore evaluated the incidence and risk factors for BP drop during IV administration of the drug.

Materials and methods: We reviewed the medical records of children with hemato-oncologic disease who were treated with IV itraconazole from January 2012 to December 2013. By analyzing systolic BP (SBP) measurements made from 4 hours before through to 4 hours after itraconazole administration, we evaluated the changes in SBP and the risk factors for an SBP drop, especially clinically meaningful ( $\geq 20 \%)$ drops.

Results: Itraconazole was administered 2,627 times to 180 patients. The SBP during the 4 hours following itraconazole administration was lower than during the 4 hours before administration $(104$ [53.0-160.33 mmHg] versus 105 [59.8-148.3 mmHg]; $P<0.001)$. The decrease in SBP was associated with the application of continuous renal replacement therapy (CRRT) $(P=0.012)$ and the use of inotropic $(P=0.005)$ and hypotensive drugs $(P=0.021)$. A clinically meaningful SBP drop was seen in $5.37 \%$ (141 out of 2,627 ) of the administrations, and the use of inotropics (odds ratio [OR] 6.70, 95\% confidence interval [CI] 3.22-13.92; $P<0.001$ ), reducing the dose of inotropics (OR 8.08; 95\% CI 1.39-46.94; $P=0.02$ ), CRRT (OR 3.10, 95\% CI 1.41-6.81; $P=0.005$ ), and bacteremia (OR $2.70,95 \%$ CI $1.32-5.51 ; P=0.007)$ were risk factors, while age was a protective factor (OR $0.93,95 \%$ CI $0.89-0.97 ; P<0.001$ ).

Conclusion: A decrease in SBP was associated with IV administration of itraconazole. It was particularly significant in younger patients with bacteremia using inotropic agents and during application of CRRT. Careful attention to hypotension is warranted during IV administration of itraconazole in this group of patients.

Keywords: itraconazole, blood pressure, hemato-oncologic disease, hypotensive drug, inotropics, CRRT

\section{Introduction}

Advances in the management of patients with hematologic malignancies over the last several decades have led to improved survival rates. The most important problem encountered in survivors is the risk of infection that follows from immunosuppression, and mortality, especially from invasive fungi, is considerable. ${ }^{1-8}$ Because it is difficult to identify the infective organisms, watchfulness and prevention are important. Amphotericin B, a classic antifungal agent, has been used for decades to treat systemic fungal infections, and various other antifungal agents with reduced toxicity have since been introduced. Itraconazole, a synthetic triazole that covers a broad spectrum of fungi, including Candida and Aspergillus spp., was approved in 1992. ${ }^{9}$ With reports 
of mostly minor adverse effects, such as headaches, minor gastrointestinal disorders, injection-site reactions, and vascular disorders, itraconazole is considered safe and effective. Currently, it is widely used for hematologic malignancy patients with prolonged neutropenic fever. ${ }^{8,10-12}$

A case of anaphylactic shock during long-term intravenous (IV) administration of itraconazole, however, has been reported, ${ }^{13}$ and an animal study revealed a negative inotropic effect proportional to the serum concentration of the drug. ${ }^{14}$ In our clinic, we have occasionally observed cases of blood pressure (BP) drop after IV administration of itraconazole. Some patients never recovered from the hypotension, and two died.

In this paper, we investigated the relationship between BP drop and IV administration of itraconazole in pediatric hemato-oncologic patients and the risk factors associated with the BP drop.

\section{Materials and methods Selection of patients}

This was a retrospective study based on the medical records of pediatric patients admitted between January 1, 2012 and December 31, 2013 to the Hemato-Oncology Department of Seoul National University Children's Hospital, a tertiary 380-bed university hospital. Hemato-oncologic patients $\leq 18$ years old who were being administered IV itraconazole (Sporanox ${ }^{\circledR}$ injection $10 \mathrm{mg} / \mathrm{mL}$; Janssen Pharmaceutica, Beerse, Belgium) prophylactically for fungal infection were enrolled. The dosage was $5 \mathrm{mg} / \mathrm{kg}$ every 12 hours for four doses, then $5 \mathrm{mg} / \mathrm{kg}$ every 24 hours. Patients were excluded if they used itraconazole therapeutically rather than prophylactically, had taken it orally, or their BP had not been measured within the 4 hours before and after the drug's administration.

The study was conducted in accordance with the Declaration of Helsinki, as amended in 2013, and was approved by the institutional review board of Seoul National University Hospital (H-1503-121-658). Written informed consent of the participants was waived.

\section{Study design and definitions}

We investigated the patients' age, sex, underlying disease, disease status at the time of itraconazole administration, and other factors that could influence BP fluctuations, namely history of taking hypotensive drugs, administration of inotropics, reduction of inotropic drug dose prior to itraconazole administration, continuous renal replacement therapy (CRRT), and the CRRT-removal rate.
Itraconazole reaches peak serum concentration in 48 hours when given orally, but immediately when given IV. ${ }^{8,15}$ To evaluate BP changes related to IV administration of itraconazole, we measured systolic BP (SBP) at least once during the 4 hours before and the 4 hours after its administration in the all patients enrolled. ${ }^{14}$

We defined baseline SBP as the mean SBP during the 4 hours before itraconazole administration and postadministration SBP as the mean SBP measurement during the 4 hours after the administration of IV itraconazole. We defined the SBP-drop rate as follows: ${ }^{16}$

$$
\underset{\text { rate }(\%)}{\text { SBP-drop }}=\frac{\text { Baseline SBP }- \text { postadministration SBP }}{\text { Baseline SBP }} \times 100
$$

We defined baseline hypotension as an SBP below the fifth percentile of the standard SBP for the age of the patient, ${ }^{17}$ and hypotensive drugs as those that could induce hypotension either by design or as an adverse side effect (ie, antihypertensive drugs, sedatives, and such opioids as fentanyl). ${ }^{16,17}$ We considered the effect of a hypotensive drug when it was given within 2 hours of its half-life. We defined systemic inflammatory response syndrome (SIRS) as having two or more findings from the following, among which, 1) or 4) had to be included: 1) body temperature $>38.5^{\circ} \mathrm{C}$ or $<36^{\circ} \mathrm{C}$; 2) mean heart rate above two standard deviations (SDs) or below the tenth percentile for age; 3) mean respiratory rate above two SDs for age; and 4) increased or decreased leukocyte count from the reference value for age ${ }^{18}$ Reference body temperature was the mean axillary temperature during the 4 hours before and the 4 hours after itraconazole administration. We defined clinically meaningful drop in SBP as an SBP drop $\geq 20 \%$ of the baseline SBP. ${ }^{19}$ In cases with a clinically meaningful drop in SBP, immediate intervention, such as IV fluids or inotropics, might be needed. Therefore, we compared the difference between the baseline SBP measured during the 4 hours before itraconazole administration and the lowest SBP measured during the 4 hours after its administration. ${ }^{15}$ We defined reduction in inotropics (drugs, including catecholamine, that can increase cardiac contractility) as a decrease in dose within the time of its activity ( 10 minutes for dopamine, 20 minutes for dobutamine, 1 hour for epinephrine, 3 minutes for norepinephrine). ${ }^{20} \mathrm{We}$ defined neutropenia as an absolute neutrophil count $<1,000 / \mu \mathrm{L}$ and bacteremia as the presence of identified organisms in blood cultures.

We classified patients' underlying diseases into the following five categories: acute leukemia, lymphoma, solid tumor, other hematologic disease (aplastic anemia, hemophagocytic 
Table 2 Systolic blood pressure according to the time related to itraconazole administration

\begin{tabular}{ll}
\hline $\begin{array}{l}\text { Time relative to itraconazole } \\
\text { administration }\end{array}$ & $\begin{array}{l}\text { Systolic blood } \\
\text { pressure }(\mathbf{m m H g})\end{array}$ \\
\hline Hours before & \\
4 & $105(47-165)$ \\
3 & $105(57-154)$ \\
2 & $106(50-156)$ \\
I & $105(56-157)$ \\
Hours after & $102(30-148)$ \\
I & $103(54-170)$ \\
2 & $104(50-160)$ \\
3 & $104(40-157)$ \\
4
\end{tabular}

Note: Data expressed as median (range).

administration than those who were not. Having SIRS or bacteremia, on the other hand, did not have a statistically significant influence on itraconazole-induced drop in SBP.

In multivariate analysis, three clinical factors were significant risk factors for a drop in SBP after itraconazole administration: administration of inotropics, administration of hypotensive drugs, and application of CRRT. Baseline hypotension, in contrast, was associated with a significantly lesser drop of SBP following itraconazole administration (Table 3).

\section{Risk factors for a $\geq 20 \%$ SBP drop}

Of the 2,627 times that itraconazole was administered, a clinically meaningful $(\geq 20 \%)$ drop in SBP followed 141

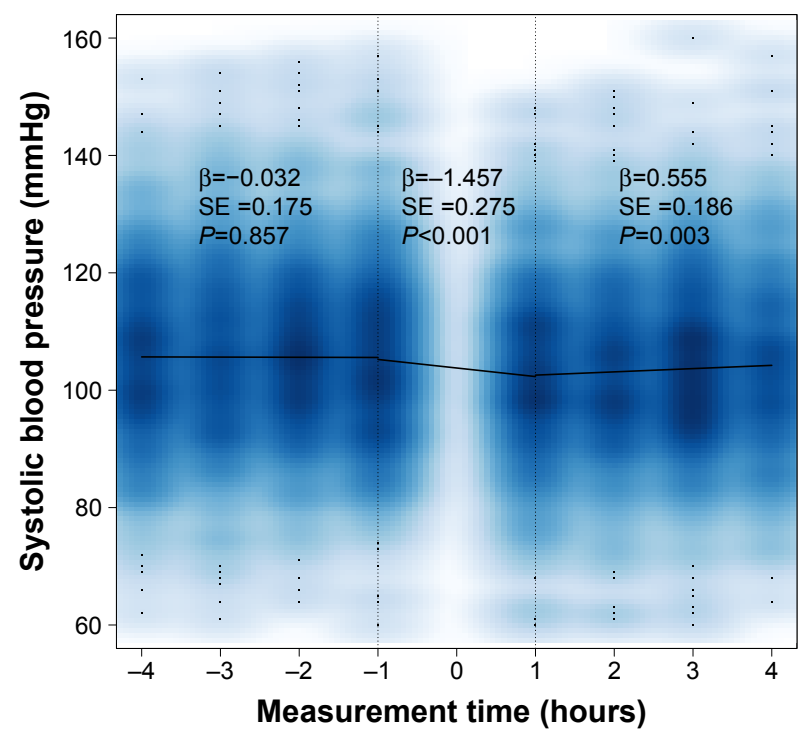

Figure I Systolic blood pressure for the 4 hours before and the 4 hours after intravenous administration of itraconazole.

Notes: Before the administration of itraconazole, there was no change in systolic blood pressure with time. After the administration of itraconazole, there was a decrease in systolic blood pressure, and it was lowest at I hour after administration. Then, there was an increasing trend in systolic blood pressure during 4 hours after the administration of itraconazole, which was statistically significant.

Abbreviation: SE, standard error. times $(5.37 \%)$. In univariate analysis of patient clinical factors, application of CRRT, administration of inotropics, reduction of inotropic dose, and bacteremia were associated with an SBP drop $>20 \%$, and increasing age reduced the risk of SBP drops $\geq 20 \%$. In multivariable analysis adjusted for potentially confounding variables, application of CRRT (odds ratio [OR] 3.10, 95\% confidence interval [CI] 1.41-6.81; $P=0.005$ ), use of inotropics (OR 6.69, 95\% CI 3.22-13.92; $P<0.001$ ), reduction of inotropic dose (OR $8.08,95 \%$ CI 1.39-46.94; $P=0.02$ ), and bacteremia (OR 2.70, $95 \%$ CI $1.32-5.51 ; P=0.007$ ) were associated with a clinically meaningful SBP drop following itraconazole administration. A 1-year increase in age, however, was associated with a significant decrease in risk (OR, 0.93, 95\% CI 0.89-0.97; $P<0.001$ ) (Table 4).

\section{Discussion}

The objectives of our study were to evaluate the relationship between the IV administration of itraconazole and the drop in BP, and what factors are associated with that relationship, especially with the clinically meaningful drop in BP. There was a statistically significant drop in SBP after the administration of itraconazole, and the rate of SBP drop was $1.46 \%$. Clinically meaningful drops in SBP were seen in $5.37 \%$ cases of 2,627 times of administration in 180 children. The risk factors contributing to the SBP drop after the administration of itraconazole were administration of inotropics, administration of hypotensive drugs, and application of CRRT. Interestingly, SIRS and bacteremia did not show statistically significant relationship with the drop in SBP. Multivariate analysis revealed that application of CRRT, use of inotropics, reduction of inotropic dose, bacteremia, and younger age were independent risk factors for the clinically meaningful drop in SBP after IV administration of itraconazole.

Patients with prolonged neutropenia who are using antifungal agents usually have multiple comorbidities, so it has been difficult to determine which factors cause drops in BP. Generally, sepsis progression is first suspected, but here we showed that SIRS and bacteremia were not risk factors for the BP drop that often follows itraconazole administration. Hypotensive drugs, such as amlodipine, nicardipine, nifedipine, nitroglycerin, labetalol, atenolol, carvedilol, losartan, and fentanyl, can lower SBP, and itraconazole was administered during the initial half-life of such drugs, which could have exacerbated the hypotension. The itraconazoleinduced increase in SBP in patients who had hypotension prior to itraconazole administration could have been due to associated interventions, such as fluid challenges or increases 
Table 3 Clinical characteristics affecting systolic blood pressure following itraconazole administration

\begin{tabular}{|c|c|c|c|c|c|c|}
\hline \multirow[t]{2}{*}{ Clinical characteristics } & \multicolumn{3}{|c|}{ Univariate analysis } & \multicolumn{3}{|c|}{ Multivariate analysis } \\
\hline & $\beta$-coefficient & SE & $P$-value & $\beta$-coefficient & SE & $P$-value \\
\hline Age (years) & -0.0427 & 0.0493 & 0.387 & & & \\
\hline \multicolumn{7}{|l|}{ Sex } \\
\hline Female & Reference & & & & & \\
\hline Male & 0.0497 & 0.5064 & 0.922 & & & \\
\hline Itraconazole dose (mg/kg) & 0.3410 & 0.4840 & 0.480 & & & \\
\hline \multicolumn{7}{|l|}{ Underlying disease } \\
\hline Acute leukemia & Reference & & & & & \\
\hline Lymphoma & 0.9250 & 1.1660 & 0.428 & & & \\
\hline Solid tumor & 0.7900 & 0.5390 & 0.143 & & & \\
\hline Other hematologic disease $\mathrm{a}^{\mathrm{a}}$ & 1.6280 & 1.0110 & 0.107 & & & \\
\hline Other disease $\mathrm{b}^{\mathrm{b}}$ & -0.4090 & 1.3080 & 0.754 & & & \\
\hline \multicolumn{7}{|l|}{ Status of therapy } \\
\hline Prechemotherapy & Reference & & & & & \\
\hline First-line chemotherapy & -0.3744 & 1.4782 & 0.800 & & & \\
\hline Postautologous SCT & -0.1399 & 1.4756 & 0.924 & & & \\
\hline Postallogeneic SCT & 0.1294 & 1.6489 & 0.937 & & & \\
\hline SIRS & 0.3460 & 0.4920 & 0.482 & & & \\
\hline Duration of underlying disease (years) & $-0.000 \mathrm{I}$ & 0.0003 & 0.710 & & & \\
\hline State of CRRT application & 3.4840 & 1.1680 & 0.003 & 3.0610 & 1.2190 & 0.0120 \\
\hline Increased removal rate of CRRT & -4.0900 & 3.8000 & 0.280 & & & \\
\hline Use of inotropics & 2.5620 & 1.0370 & 0.014 & 2.6970 & 0.9560 & 0.0048 \\
\hline Reduced inotropics & 5.4000 & 3.7400 & 0.149 & & & \\
\hline Use of hypotensive drugs & 1.8000 & 0.7010 & 0.010 & 1.6060 & 0.6940 & 0.0207 \\
\hline Bacteremia & 0.9000 & 1.0610 & 0.400 & & & \\
\hline Baseline hypotension & -11.1890 & 1.4510 & $<0.001$ & -11.7380 & 1.4060 & $<0.001$ \\
\hline
\end{tabular}

Notes: a Other hematologic diseases: aplastic anemia, hemophagocytic lymphohistiocytosis, Langerhans cell histiocytosis, Fanconi anemia, idiopathic thrombocytopenic purpura, myelodysplastic syndrome, and thrombotic thrombocytopenic purpura. ${ }^{\circ}$ Other diseases: chronic granulomatous disease, severe combined immunodeficiency, and osteopetrosis.

Abbreviations: SE, standard error; SCT, stem cell transplantation; SIRS, systemic inflammatory response syndrome; CRRT, continuous renal replacement therapy.

in inotropic dose during itraconazole administration. We therefore cannot exclude the possibility of underestimating the severity of the SBP drop in this patient group, and it is difficult to rule out baseline hypotension as a possible risk factor for SBP drop after itraconazole administration. Younger age was a risk factor for a clinically meaningful SBP drop associated with itraconazole, so further studies are needed to clarify the relationship between age and the effect of itraconazole on BP.

The use of inotropics and reducing their dose were risk factors for meaningful SBP drops. Itraconazole itself has negative inotropic effects in rabbits, dogs, ${ }^{14,21}$ and healthy human volunteers, and administration of itraconazole to patients with congestive heart failure requires great care..$^{21,22}$ Because itraconazole administered IV reaches peak serum concentration rapidly compared with the oral form, ${ }^{8}$ its negative inotropic effects appear more abruptly. In patients who maintain their cardiac contractility and SBP in the presence of inotropics, IV administration of itraconazole can offset the effect of inotropics and cause an SBP drop. Reducing the inotropic dose was not associated with a drop in SBP, but was a risk factor for a clinically meaningful drop in SBP. We speculate that the negative inotropic effect of itraconazole is more prominent in a specific group of patients in whom the SBP drop can be more severe. The application of CRRT and an increase in its removal rate were also risk factors for a clinically meaningful SBP drop. CRRT usually reduces the risk of hemodynamic instability better than conventional hemodialysis, but we think that the negative inotropic effect of itraconazole is more prominent in this patient group.

Our study has several limitations. It was a single-center experience. We analyzed only SBP, so the effect of itraconazole on diastolic BP could not be evaluated. Also, since this was a retrospective study, we could not calculate the glomerular filtration rate precisely, and thus could not exclude the possibility that CRRT affected the serum level of itraconazole.

In light of our findings, we recommend that BP be measured more frequently than at the usual 4- to 8-hour intervals in patients being administered IV itraconazole. We also recommend that itraconazole be used with caution in patients taking hypotensive or inotropic drugs and 


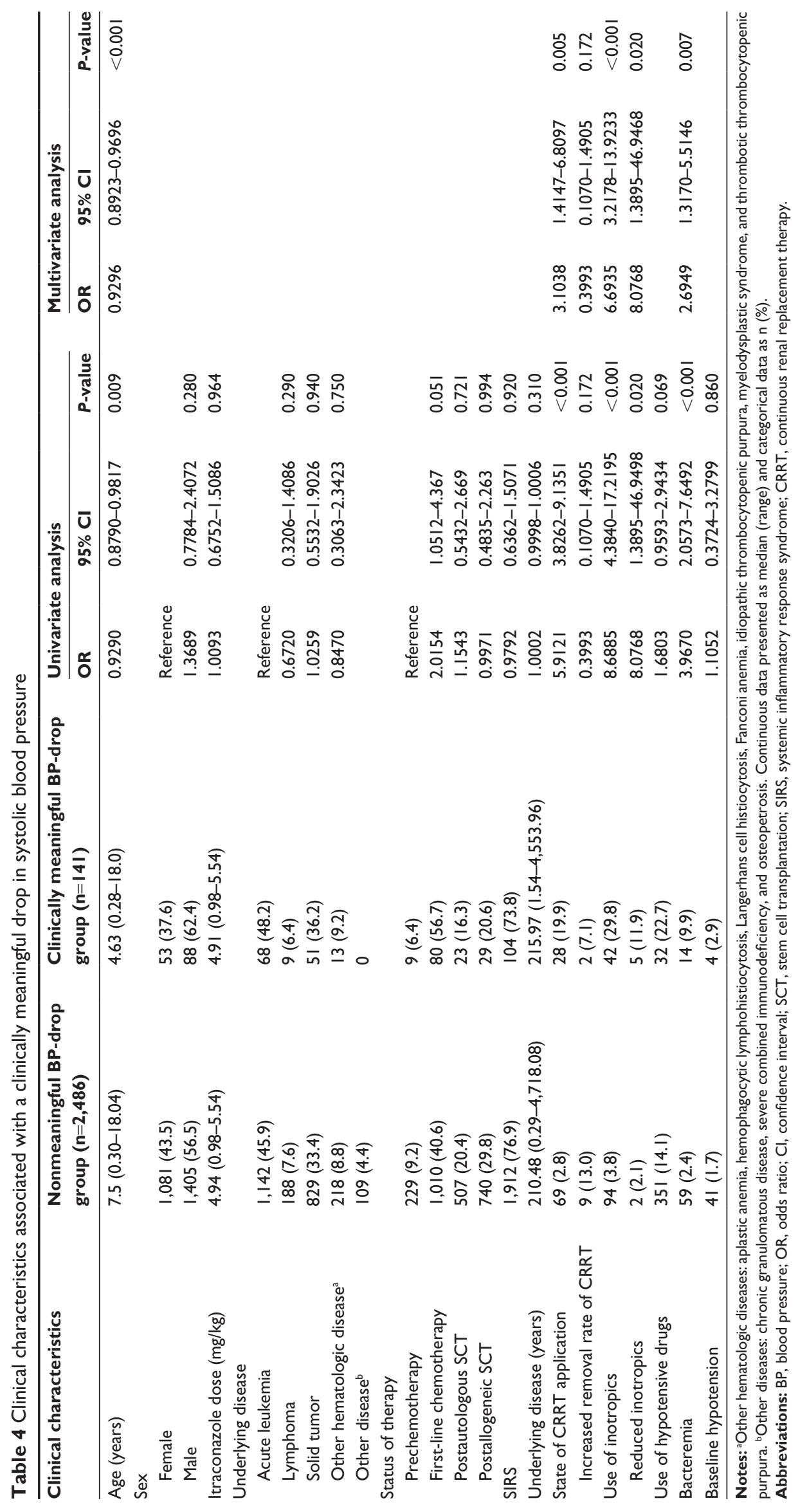


when applying CRRT, especially in younger age groups. In addition, a prospective, age-stratified study to evaluate more precisely the relationship between IV itraconazole and BP drop is needed.

\section{Disclosure}

The authors report no conflicts of interest in this work.

\section{References}

1. Wingard JR. Empirical antifungal therapy in treating febrile neutropenic patients. Clin Infect Dis. 2004;39 Suppl 1:S38-S43.

2. Zaoutis TE, Argon J, Chu J, Berlin JA, Walsh TJ, Feudtner C. The epidemiology and attributable outcomes of candidemia in adults and children hospitalized in the United States: a propensity analysis. Clin Infect Dis. 2005;41(9):1232-1239.

3. Zaoutis TE, Heydon K, Chu JH, Walsh TJ, Steinbach WJ. Epidemiology, outcomes, and costs of invasive aspergillosis in immunocompromised children in the United States, 2000. Pediatrics. 2006;117(4): e711-e716.

4. Kobayashi R, Suzuki D, Yasuda K, Kobayashi K. Itraconazole for invasive fungal infection with pediatric malignancies. Pediatr Int. 2010; 52(5):707-710.

5. Prasad PA, Coffin SE, Leckerman KH, Walsh TJ, Zaoutis TE. Pediatric antifungal utilization: new drugs, new trends. Pediatr Infect Dis $J$. 2008; 27(12):1083-1088.

6. Steinbach WJ. Pediatric aspergillosis: disease and treatment differences in children. Pediatr Infect Dis J. 2005;24(4):358-364.

7. Zaoutis TE, Coffin SE, Chu JH, et al. Risk factors for mortality in children with candidemia. Pediatr Infect Dis J. 2005;24(8):736-739.

8. Kim H, Shin D, Kang HJ, et al. Successful empirical antifungal therapy of intravenous itraconazole with pharmacokinetic evidence in pediatric cancer patients undergoing hematopoietic stem cell transplantation. Clin Drug Investig. 2015;35(7):437-446.

9. Lewis RE. Current concepts in antifungal pharmacology. Mayo Clin Proc. 2011;86(8):805-817.

10. Ohta K, Nishiki Kosaka S, Nakao Y, et al. Efficacy and safety of intravenous itraconazole as empirical antifungal therapy for persistent fever in neutropenic patients with hematological malignancies in Japan. Int J Hematol. 2009;89(5):649-655.
11. Cheng S, Zhou JF, Zou P, Huang XJ, Jin J, Shen ZX. Efficacy and safety of itraconazole as empirical antifungal therapy in febrile neutropenic patients with hematologic malignancies: an open-lable [sic], multicenter, observational trial in a Chinese cohort. Chin Med J (Engl). 2011; 124(22):3670-3675.

12. Abdel-Rahman SM, Jacobs RF, Massarella J, et al. Single-dose pharmacokinetics of intravenous itraconazole and hydroxypropyl- $\beta$-cyclodextrin in infants, children, and adolescents. Antimicrob Agents Chemother. 2007;51(8):2668-2673.

13. Chen J, Song X, Yang P, Wang J. Appearance of anaphylactic shock after long-term intravenous itraconazole treatment. Ann Pharmacother. 2009;43(3):537-541.

14. Qu Y, Fang M, Gao B, et al. Itraconazole decreases left ventricular contractility in isolated rabbit heart: mechanism of action. Toxicol Appl Pharmacol. 2013;268(2):113-122.

15. Willems L, van der Geest R, de Beule K. Itraconazole oral solution and intravenous formulations: a review of pharmacokinetics and pharmacodynamics. J Clin Pharm Ther. 2001;26(3):159-169.

16. Kleinman ME, Chameides L, Schexnayder SM, et al. Part 14: Pediatric advanced life support: 2010 American Heart Association Guidelines for Cardiopulmonary Resuscitation and Emergency Cardiovascular Care. Circulation. 2010;122(18 Suppl 3):S876-S908.

17. Degoute CS. Controlled hypotension: a guide to drug choice. Drugs. 2007;67(7):1053-1076.

18. Goldstein B, Giroir B, Randolph A. International pediatric sepsis consensus conference: definitions for sepsis and organ dysfunction in pediatrics. Pediatr Crit Care Med. 2005;6(1):2-8.

19. Nafiu OO, Voepel-Lewis T, Morris M, et al. How do pediatric anesthesiologists define intraoperative hypotension? Paediatr Anaesth. 2009; 19(11):1048-1053.

20. Taketomo CK. Pediatric \& Neonatal Dosage Handbook. 18th ed. Hudson, OH: Lexi-Comp; 2011.

21. McKee J, Rabinow B, Cook C, Gass J. Nanosuspension formulation of itraconazole eliminates the negative inotropic effect of Sporanox in dogs. J Med Toxicol. 2010;6(3):331-336.

22. Ahmad SR, Singer SJ, Leissa BG. Congestive heart failure associated with itraconazole. Lancet. 2001;357(9270):1766-1767.

\section{Publish your work in this journal}

Drug Design, Development and Therapy is an international, peerreviewed open-access journal that spans the spectrum of drug design and development through to clinical applications. Clinical outcomes, patient safety, and programs for the development and effective, safe, and sustained use of medicines are a feature of the journal, which

\section{Dovepress}

has also been accepted for indexing on PubMed Central. The manuscript management system is completely online and includes a very quick and fair peer-review system, which is all easy to use. Visit http://www.dovepress.com/testimonials.php to read real quotes from published authors. 